\title{
Interplay between Hydration Water and Headgroup Dynamics in Lipid Bilayers
}

\author{
P. Berntsen, ${ }^{*,+}$ C. Svanberg, ${ }^{\neq}$and J. Swenson ${ }^{*,+}$ \\ ${ }^{\dagger}$ Department of Applied Physics, Chalmers University of Technology, SE-412 96 Göteborg, Sweden \\ ${ }^{\ddagger}$ Borealis Innovation Center, Industrivägen, SE-444 86 Stenungsund, Sweden
}

ABSTRACT: In this study, the interplay between water and lipid dynamics has been investigated by broadband dielectric spectroscopy and modulated differential scanning calorimetry (MDSC). The multilamellar lipid bilayer system 1,2-dimyristoyl-sn-glycero-3-phosphocholine (DMPC) has been studied over a broad temperature range at three different water contents: about 3, 6, and 9 water molecules per lipid molecule. The results from the dielectric relaxation measurements show that at temperatures $<250 \mathrm{~K}$ the lipid headgroup rotation is
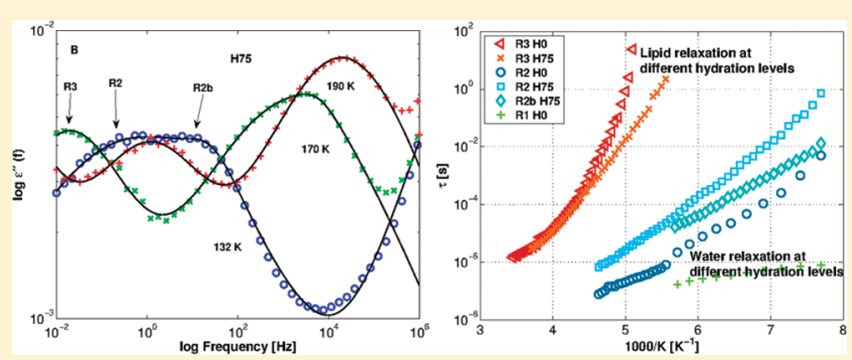
described by a super-Arrhenius temperature dependence at the lowest hydration level and by the Arrhenius law at the highest hydration level. This difference in the temperature dependence of the lipid headgroup rotation can be explained by the increasing interaction between the headgroups with decreasing water content, which causes their rotational motion to be more cooperative in character. The main water relaxation shows an anomalous dependence on the water content in the supercooled and glassy regime. In contrast to the general behavior of interfacial water, the water dynamics is fastest in the driest sample and its temperature dependence is best described by a super-Arrhenius temperature dependence. The best explanation for this anomalous behavior is that the water relaxation becomes more determined by fast local lipid motions than by the intrinsic water dynamics at low water contents. In support for this interpretation is the finding that the relaxation time of the main water process is faster than that in most other host systems at temperatures below $180 \mathrm{~K}$. Thus, the dielectric relaxation data show clearly the strong interplay between water and lipid dynamics; the water influences the lipid dynamics and vice versa. In the MDSC data, we observe a weak enthalpy relaxation at $203 \mathrm{~K}$ for the driest sample and at $179 \mathrm{~K}$ for the most hydrated sample, attributed to the freezing-in of the lipid headgroup rotation observed in the dielectric data, since this motion reaches a time scale of about $100 \mathrm{~s}$ at about the same temperatures.

\section{INTRODUCTION}

Biomembranes, phospholipid bilayers that form the boundaries of cells, exhibit a fluidity that is dependent on the amount of hydration water. ${ }^{1,2}$ Of particular interest are the viscoelastic properties of biomembranes, as they have consequences for the morphology and dynamics of the cell. ${ }^{3,4}$ While biological function is primarily associated with the fully hydrated form of biological membranes, the less hydrated states are highly relevant for cryopreservation of biological membranes and cells, which is of practical and industrial importance in, e.g., clinical medicine, pharmacology, food science, ${ }^{5}$ and the preservation of embryos for test tube fertilization. ${ }^{6}$

Investigations of the dehydrated states give us the possibility to better understand how water affects its nearest surroundings and the interplay between water molecules and the biological surface. Because of the complexity of biological membranes, simpler model membranes containing one type of phospholipid molecule or a simple mixture of lipid molecules are generally studied. An example of such phospholipids is phosphatidycholine which consists of a polar headgroup region and hydrophobic acyl chains, which together are the driving force for self-assembly into multilamellar structures consisting of lipid bilayers and interlayer water. $^{7}$

The dynamics of model membranes of phospholipids and the associated water has been probed by experimental techniques such as NMR, ${ }^{8}$ quasi-elastic neutron scattering (QENS), ${ }^{9-11}$ dynamic light scattering, ${ }^{12}$ dielectric spectroscopy, ${ }^{13-21}$ and ultrasonic spectroscopy. ${ }^{22}$ Computer simulations have also improved our understanding of the structure and dynamics at different hydration levels, ${ }^{2}$ temperatures, and phase configurations ${ }^{23,24}$ and of the structural and dynamical hydrogen bonding features at the lipid-water interface. ${ }^{25}$ These studies highlight the wide variation in dynamics of hydrated lipid membranes that include several different types of processes, such as fast diffusion of water molecules, rotation of the lipid heads, segmental motion of the lipid tails, and 2D translational diffusion of lipids within the layers. A full understanding of the important relation between lipid and solvent

Received: November 15, 2010

Revised: January 11, 2011

Published: February 8, 2011 


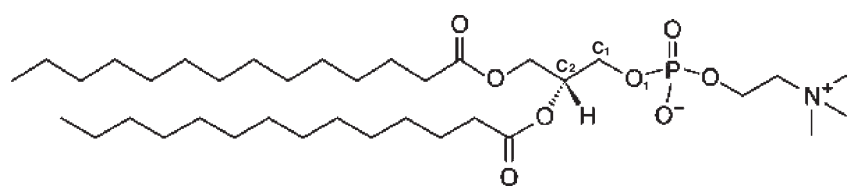

Figure 1. Schematic picture of the 1,2-dimyristoyl-sn-glycero-3-phosphocholine (DMPC) molecule.

dynamics from the glassy state up to ambient temperatures is, however, lacking.

In this work, we have used broadband dielectric spectroscopy (BDS) and modulated differential scanning calorimetry (MDSC) to study the water and lipid dynamics of multilamellar membranes at low hydration levels from glassy temperatures up to physiological temperatures. The phospholipid we have used is dimyristoylphosphocholine (DMPC) (see Figure 1). It is naturally found in biological membranes such as the cellular plasma membrane and consists of two saturated $\mathrm{C}_{14}$ carbon acids connected to a dipolar headgroup. ${ }^{26}$ Dielectric spectroscopy is a well suited method for the investigation of polar materials, and it covers a large dynamical range from ps up to several minutes. It provides important dynamical information, such as relaxation times and activation energies of relaxation and charge transport processes. MDSC measurements provide information of thermal events as a function of time and temperature in materials which involve endothermic or exothermic processes, or changes in heat capacity. Here, we focus on the water and lipid dynamics at low temperatures, since it is of importance to explore the nature of a possible glass-like behavior of lipid membranes and to what extent water affects this behavior. Moreover, it is of interest to extend previous dielectric measurements on supercooled water in different types of host materials ${ }^{21,27-30}$ to understand how the dynamics of supercooled confined water depends on the structure and the hydrophilic/hydrophobic character of its nearest environment. ${ }^{31,32}$

\section{— EXPERIMENTAL METHODS}

To achieve a supported phospholipid multibilayer system, the approach described in ref 33 was followed. A $10 \mathrm{mg}$ portion of 1,2-dimyristoyl-sn-glycero-3-phosphocholine, molecular weight 677.93 (DMPC, Avanti Polar Lipids), was dissolved in $1 \mathrm{~mL}$ of isopropanol. Repeatedly, $0.05-0.1 \mathrm{~mL}$ of the suspension was deposited until the whole suspension had evaporated on cleaned brass electrodes for the dielectric measurements and in a pan for the MDSC measurements. The membrane films were tempered around the gel-fluid transition temperature to anneal defects and to get a better alignment of the lipids. To remove residual solvent, the samples were placed under a vacuum $\left(10^{-3} \mathrm{mbar}\right)$ for 2-3 h. Partially hydrated DMPC multilayers were prepared through vapor phase equilibrium, by storing the vacuum pumped samples at $298 \mathrm{~K}$ in sealed desiccators with saturated Milli-Q water salt solutions for 2-3 days. Relative humidities were $75 \%(\mathrm{NaCl})$ and $84 \%(\mathrm{KCl})^{34}$ and water absorption 0.08 and $0.16 \mathrm{~g}$ of water/g of lipid. A basically dry sample was also prepared by putting the sample under a vacuum overnight. The samples are referred to as H0, H75, and H84 in the text, and they contain approximately 3, 6, and 9 water molecules per lipid molecule, respectively. Thus, even the vacuum-dried HO sample contains some base water. These water molecules are hydrogen bonded to lipid oxygen atoms and diffuse slowly between the oxygen atoms of the phosphate and the oxygen atoms double bonded to carbon atoms (see Figure 1). ${ }^{25}$
Additional hydration water is located around the choline group $-\mathrm{N}\left(\mathrm{CH}_{3}\right)_{3}$ (see Figure 1) for moderate hydration levels and finally in the interlayer region for the highest hydration levels. ${ }^{25,35}$

MDSC Measurements. The modulated differential scanning calorimetry (MDSC) experiments were performed using a TA Q1000 thermal analyzer. This technique is an extension of conventional DSC, where a sinusoidal wave modulation is superpositioned on a linear temperature ramp; see, e.g., ref 36 . This enables a separation of thermal events and transitions into "kinetic" and "heat capacity" responses of the measured sample. The reversing component in MDSC experiment is related to the sample's heat capacity; a glass transition event is detected in the reversing heat flow. On the other hand, processes such as enthalpic relaxation, crystallization, evaporation, decomposition, and cure are resolved into the kinetic component or nonreversing heat flow. For a melting transition, the interpretation is not straightforward. Melting can occur in the reversing heat flow as well as in the nonreversing heat flow, and moreover, the relative contribution of these components depends on the experimental conditions (underlying heating rate, modulation period, modulation amplitude, sample thickness). Hermetically sealed pans were used for the samples and with an empty pan as the reference. The MDSC experiments were performed in a heat only temperature-modulation procedure starting at $100 \mathrm{~K}$ and then heated to $283 \mathrm{~K}$, with a ramp rate of $2 \mathrm{~K} / \mathrm{min}$ and a temperature modulation of $\pm 0.32 \mathrm{~K}$ every $60 \mathrm{~s}$. The sample pan was quenched in liquid nitrogen before the MDSC heating scan was started.

Dielectric Relaxation Measurements. The experiments were performed using an Alpha-S high resolution dielectric spectrometer from Novocontrol over the frequencies $10^{-2}-10^{7} \mathrm{~Hz}$ using electrodes with a diameter of $20 \mathrm{~mm}$. Isothermal frequency scans of the complex dielectric function $\varepsilon^{*}(\omega)=\varepsilon^{\prime}(\omega)-i \varepsilon^{\prime \prime}(\omega)$ were performed from 130 up to $310 \mathrm{~K}$ in steps of 2 or $5 \mathrm{~K}$, depending on the temperature. Prior to the dielectric experiments, the samples were quenched down to $77 \mathrm{~K}$ by submerging them into a liquid nitrogen bath in order to minimize crystallization of the water. The exact thickness of each sample is not known, and therefore, we will refrain from discussing the amplitudes of the processes.

One conductivity/polarization term and a sum of HavriliakNegami functions were used to fit the imaginary part of the dielectric function $\varepsilon^{\prime \prime}(\omega)$ :

$$
\varepsilon^{\prime \prime}(\omega)=\left(\frac{\sigma}{\varepsilon_{0} \omega}\right)^{s}+\sum \operatorname{Im}\left[\frac{\Delta \varepsilon}{\left(1+(i \omega \tau)^{\alpha}\right)^{\beta}}\right]
$$

The first term on the right-hand side of eq 1 quantifies the dc conductictivity and polarization effects in terms of $\varepsilon^{\prime \prime}(\omega)$ by the fit parameters $\sigma$ and $s ; s=1$ for pure dc conductivity and $s<1$ when also polarization effects contribute. The conductivity term is visible as an increase in $\varepsilon^{\prime \prime}$ toward low frequencies. $\varepsilon_{0}$ is the vacuum permittivity. $\tau$ is the relaxation time (the inverse of the relaxation rate), and $\Delta \varepsilon$ is the relaxation strength of a given relaxation process. The exponents $\alpha$ and $\beta[0<\alpha, \beta \leq 1]$ define the symmetrical and asymmetrical broadening, respectively, of the dielectric loss peaks compared to a Debye peak $[\alpha=$ $\beta=1]$.

The main structural relaxation, or the so-called $\alpha$ process in viscous liquids, is closely linked to the glass transition and has a non-Arrhenius temperature dependence that is generally 
described by the Vogel-Fulcher-Tamman equation ${ }^{37-39}$

$$
\tau=\tau_{0} \exp \left(\frac{D T_{0}}{T-T_{0}}\right) \quad\left(T_{0}<T\right)
$$

This equation gives an activation energy which increases on cooling, i.e., a super-Arrhenius temperature dependence, and a relaxation time which becomes infinite at $T_{0} . \tau_{0}$ is the relaxation time in the high temperature limit, usually $10^{-14}-10^{-13} \mathrm{~s}$, corresponding to a typical molecular vibration time. $D$ is the so-called fragility index which determines the deviation from an Arrhenius temperature dependence. A smaller $D$ value corresponds to a larger deviation from the well-known Arrhenius behavior

$$
\tau=\tau_{0} \exp \left(\frac{E}{k_{\mathrm{b}} T}\right)
$$

which gives the relaxation time at the temperature $T$ by $\tau_{0}$ and the activation energy $E$ of an ordinary thermally activated process. The Arrhenius law is commonly used to describe the temperature dependence of noncooperative secondary $(\beta)$ relaxations. ${ }^{40}$

\section{RESULTS}

Thermal Events with MDSC. In Figure 2, we show the MDSC data of the $\mathrm{H} 0$ and $\mathrm{H} 84$ samples, respectively. In order to have a reliable Fourier deconvolution in MDSC, one needs at least four cycles of the modulation during the transition (full width at half-maximum of the peak). ${ }^{36}$ For the narrow peaks shown in Figure 2, we are far from reaching this criterion. Therefore, we do not get reliable results in the reversing and nonreversing heat flow. However, the data for the total heat flow should be reliable and therefore used in the case of the narrow peaks.

The MDSC scan of the $\mathrm{H} 0$ sample (see Figure 2A) is rather featureless except for a small endothermic event at $203 \mathrm{~K}$ in the total heat flow, also observed in the reversing component, which is an indication that this thermal event probably is due to the same kind of enthalpy relaxation of the lipids when the time scale of the associated relaxation process reaches the experimental time scale upon reheating.

The higher hydration level of the H84 sample (see Figure 2B) results in an MDSC scan with two endotherms at 131 and $179 \mathrm{~K}$, which we interpret as enthalphy relaxations from water and lipid molecules, respectively. The weak exothermic feature at $215 \mathrm{~K}$ is likely a result of small rearrangements of amorphous structure to a crystalline structure before the main endothermic feature sets in at about $220 \mathrm{~K}$. This main event continues to $236 \mathrm{~K}$, and it fulfills the four-cycle criterion to distinguish between reversing and nonreversing signals. A small step can be observed in the heat capacity related reversing term, but the main component of this event is kinetic nonreversing, probably due to some kind of melting. The small exothermic feature at $273 \mathrm{~K}$ may be an overlapping phenomena such as a structural recovery of the lipids that follows from passing the melting of a small amount of surface water on the lipid sample. All the observed thermal events are presented in Table 1.

Dielectric Relaxation Data. Dielectric loss spectra at selected temperatures are presented in Figure 3. The dielectric loss spectra $\varepsilon^{\prime \prime}(f)$ for all hydration levels exhibit two main processes called R2, mainly due to the relaxation of water molecules, and $\mathrm{R} 3$, due to the relaxation of the lipid headgroups. The interpretations of these processes will be discussed in more detail in the Discussion section below. In the dried HO sample
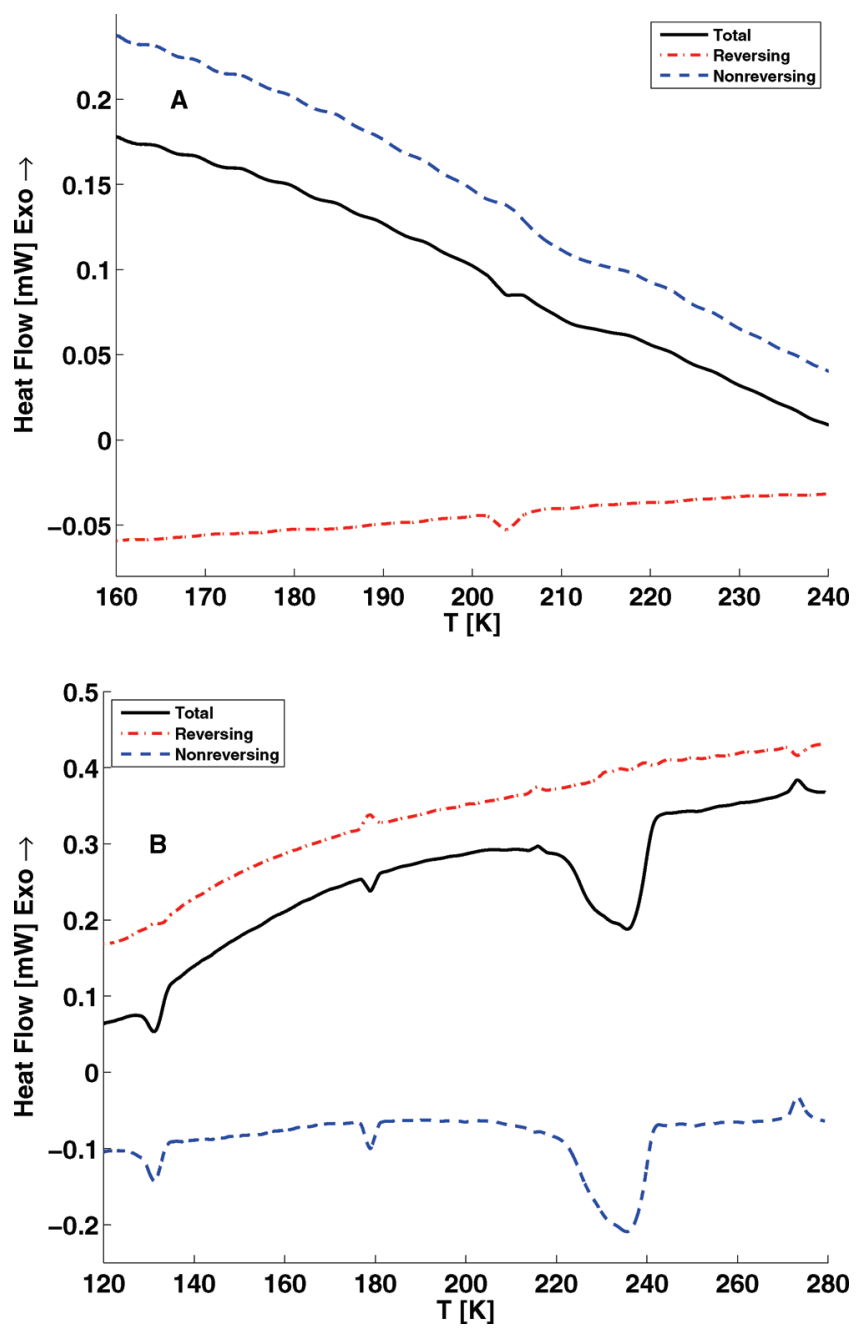

Figure 2. MDSC heating scans of the H0 (A) and H84 (B) samples. Total heat flow, black solid line; reversing heat flow, red dash-dotted line; nonreversing heat flow, blue dashed line. The thermal events are explained in the text and also given in Table 1.

(see Figure 3A), the main water relaxation ( $\mathrm{R} 2$ ) is nearly two decades faster than R2 for the hydrated samples at the lowest measured temperatures. At about $210 \mathrm{~K}$, a weaker relaxation process $\mathrm{R} 1$ merges with $\mathrm{R} 2$. We think that $\mathrm{R} 1$ is due to a local water relaxation, since a similar process has been observed in other systems containing supercooled or glassy interfacial water. ${ }^{41}$ The lipid headgroup relaxation (R3) enters the spectra at the lowest frequency at $196 \mathrm{~K}$, which is similar to the temperature (203 K) where we observed the enthalpy relaxation in the MDSC data.

In the hydrated samples H75 (see Figure 3B) and H84 (see Figure $3 \mathrm{C}$ ), a weaker water process $\mathrm{R} 2 \mathrm{~b}$ is merging with $\mathrm{R} 2$, and above $175 \mathrm{~K}$, they appear as one process. The low intensity process R1 is observed at low temperatures also in the H84 sample, where it is considerably faster than the other observed relaxation processes. At higher temperatures, $\mathrm{R} 1$ is submerged into the high frequency side of R2. R1 was obscured during measurements of the H75 sample due to problems with the dielectric signal at the highest measured frequencies. For the hydrated samples, the main water process $\mathrm{R} 2$ and the lipid relaxation $\mathrm{R} 3$ enter the low frequency side of the spectra at 130 and $170 \mathrm{~K}$, respectively, which are similar temperatures to the enthalpy relaxations we observed in the MDSC measurements. 
Table 1. Measured Thermal Events

$\begin{array}{lcl}\text { sample } & \text { temperature }(\mathrm{K}) & \\ \text { H0 } & 203 & \text { event } \\ \text { H84 } & 131 & \text { enthalpy relaxation - lipid associated } \\ & 179 & \text { enthalpy relaxation - lipid associated } \\ & 220-236 & \text { endothermic melting } \\ & 273 & \text { melting/structural recovery of the lipids }\end{array}$

Contributions from $\mathrm{dc}$ conductivity and polarization effects become visible at low frequencies as a rise in $\varepsilon^{\prime \prime}$ above $180 \mathrm{~K}$ for the hydrated samples and above $215 \mathrm{~K}$ for the almost dry (H0) sample.

Relaxation times obtained from the curve fitting with HavriliakNegami functions (eq 1) to the dielectric loss spectra $\varepsilon^{\prime \prime}(f)$, together with the temperatures of the thermal events we observed in MDSC, are presented in Figure 4. Equations 2 and 3 were used to describe the temperature dependences of the relaxation times. Fit parameters are presented in Table 2.

\section{DISCUSSION}

In Figure 5A, we compare the relaxation processes for the hydration levels $\mathrm{H0}$ and $\mathrm{H} 75$ (the results for $\mathrm{H} 84$ are almost identical to those for H75, and therefore only shown in Figure 4). Let us start to discuss process R3 and its origin. By comparing with literature data on both confined water ${ }^{29,30}$ and other lipid systems, ${ }^{2,42,43}$ we can conclude that process R3 must originate from the dynamics of the lipid molecules, as stated above. In fact, the time scale of the process is in good agreement with previous findings ${ }^{2,42,43}$ for the lipid headgroup rotation, as shown in Figure $5 \mathrm{~B}$. At the highest temperatures $(>250 \mathrm{~K})$, the lipid headgroup rotation is almost independent of the water content, but with decreasing temperature the difference increases due to an increasing super-Arrhenius (i.e., VFT) temperature dependence with decreasing hydration level. Thus, if an analogy is made to the structural $\alpha$-relaxation of a supercooled liquid, we can conclude that the fragility of the lipid headgroup rotation increases with decreasing water content (the fragility index $D$ decreases from 27.14 to 3.83 when the water content decreases from $\mathrm{H} 75$ to $\mathrm{H} 0$ ). These $D$ values are typical for fragile organic liquids, whereas nonfragile or "strong" liquids show $D$ values greater than $100 .^{40} \mathrm{~A}$ strong super-Arrhenius temperature dependence was also observed for the lipid headgroup relaxation in moderately hydrated POPC lipid multibilayers. ${ }^{42}$ However, at the highest hydration level (H84) of the present study, the superArrhenius behavior is lost and the temperature dependence of the lipid headgroup rotation is best described by the Arrhenius law, as shown in Figure 4C. It is an interesting finding that the temperature dependence of the lipid headgroup rotation is so sensitive to the hydration level. Since a super-Arrhenius behavior is generally a signature of cooperative motions occurring on long length scales, this finding suggests that the motion of different lipid headgroups becomes more correlated with decreasing water content. Thus, it seems as if the hydration water "screens" the interaction between adjacent lipid headgroups. This interpretation is supported by previous results from MD simulations, where it was found that the correlation between the positive nitrogens and the negative phosphate groups on adjacent bilayers increases with decreasing water content. ${ }^{2}$ In the case of the highest hydration level (H84), it seems to be a sufficient amount of water around the lipid headgroups to fully "screen" the
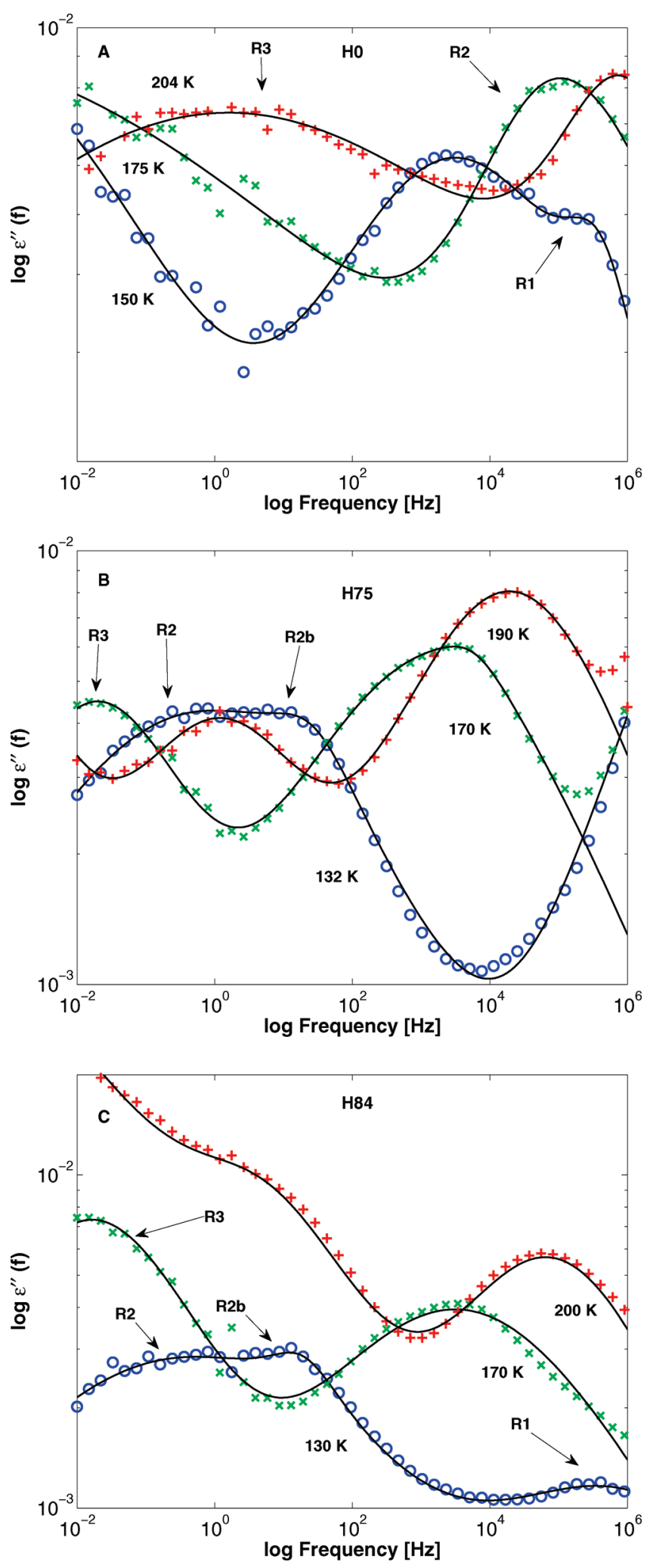

Figure 3. Dielectric loss spectra of H0 (A), H75 (B), and H84 (C) at selected temperatures. The curve fits (by eq 1 ) to the data are given by the solid lines.

interaction between adjacent lipid headgroups, thereby causing their motion to be noncooperative in character. Instead, the rotational dynamics of the lipid headgroups becomes influenced by the faster dynamics of the surrounding water molecules. This 

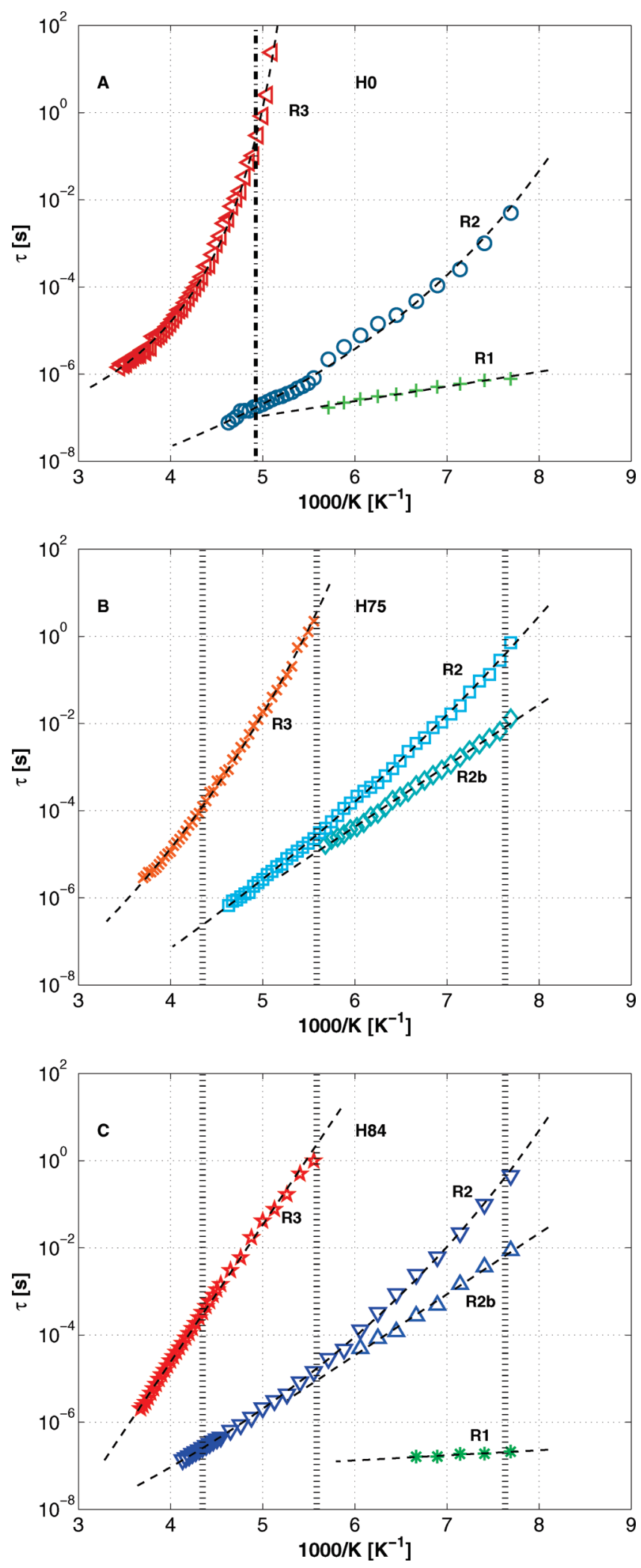

Figure 4. Arrhenius plot of the temperature dependent relaxation times obtained from the curve fitting of the dielectric loss data presented in Figure 3 showing the temperature dependences of the processes observed in the H0, H75, and H84 samples, respectively. The temperature dependences are described by eq 2 or 3 (dashed lines). Fit parameters are presented in Table 2. Vertical lines mark the thermal events observed in the MDSC measurements.
Table 2. Fit Parameters from eqs 2 and 3 Describing the Temperature Dependences of the Relaxation Times Presented in Figure 4

\begin{tabular}{lcccr} 
& $\tau_{0}$ & $E(\mathrm{eV})$ & $T_{0}(\mathrm{~K})$ & $D$ \\
& & & \\
$\mathrm{R} 1$ & $2.3 \times 10^{-9}$ & 0.07 & & \\
$\mathrm{R} 2$ & $9.0 \times 10^{-11}$ & & 77.8 & 12.13 \\
$\mathrm{R} 3$ & $7.8 \times 10^{-9}$ & & 166 & 3.83 \\
& & $\mathrm{H} 75$ & & \\
$\mathrm{R} 2$ & $7.8 \times 10^{-13}$ & & 43.7 & 53.8 \\
$\mathrm{R} 2 \mathrm{~b}$ & $1.7 \times 10^{-13}$ & 0.28 & & \\
$\mathrm{R} 3$ & $2.5 \times 10^{-12}$ & & 90.8 & 27.14 \\
& & $\mathrm{H} 84$ & & \\
$\mathrm{R} 1$ & $2.6 \times 10^{-8}$ & 0.023 & & \\
R2 & $2.5 \times 10^{-11}$ & & 67.3 & 22.3 \\
R2b & $1.4 \times 10^{-13}$ & 0.28 & & \\
R3 & $6.3 \times 10^{-18}$ & 0.62 & & \\
\hline
\end{tabular}

prominent role of the hydration water on the headgroup dynamics is supported by recent neutron scattering investigations. ${ }^{44}$

The water content of planar lipid bilayers also has a more general effect on the dynamical properties of the membrane. It has been shown ${ }^{2,45}$ that the elasticity of lipid bilayers increases with increasing hydration level. This can possibly be related to a recent finding that soft deformable colloids make strong colloidal glasses. ${ }^{46}$ The fragility of deformable colloid particles was shown to be dictated by elastic properties on the scale of individual colloidal particles. ${ }^{46}$ Thus, an analogy with colloids can be made; highly hydrated lipid membranes should behave as strong glass formers, whereas dehydrated lipid membranes should exhibit a fragile behavior; i.e., the hydration water controls both the elasticity and the fragility of the lipid bilayer systems. This is supported by the observed hydration dependence of the lipid headgroup rotation, although it is not evident that the headgroup rotation should be directly related to the glass transition of the lipid membrane. The enthalpy relaxation observed at 179 and $203 \mathrm{~K}$ in the MDSC data of the H84 and H0 samples, respectively, is likely due to the freezing-in (on a time scale of $100 \mathrm{~s}$, which is commonly used to define the dynamic glass transition) of the lipid headgroup dynamics, since this occurs at similar temperatures in the dielectric relaxation data. However, whether this enthalpy relaxation of the lipid headgroups should be associated with a true glass transition (i.e., the freezing-in of a viscosity related structural $\alpha$-relaxation) or the freezing-in of a more local relaxation process cannot be established in this study. However, a recent calorimetric study by Shalaev et al. ${ }^{47}$ suggests that the true $T_{\mathrm{g}}$ of such a low hydrated DMPC is located as high as around $340 \mathrm{~K}$, which would imply that the calorimetric $T_{\mathrm{g}}$ cannot be associated with the freezing-in of lipid headgroup dynamics. Rather, the high $T_{\mathrm{g}}$ suggests that it should be related to the freezing-in of slow cooperative motions involving several lipid molecules.

Let us now discuss the observed water dynamics in the lipid bilayers. Due to the large dielectric constant of water, dielectric processes due to reorientational motions of water molecules are generally easily observed even for a very small fraction of water in the system. Therefore, we interpret R2 and R2b to be caused by the main relaxation of water molecules with slightly different 

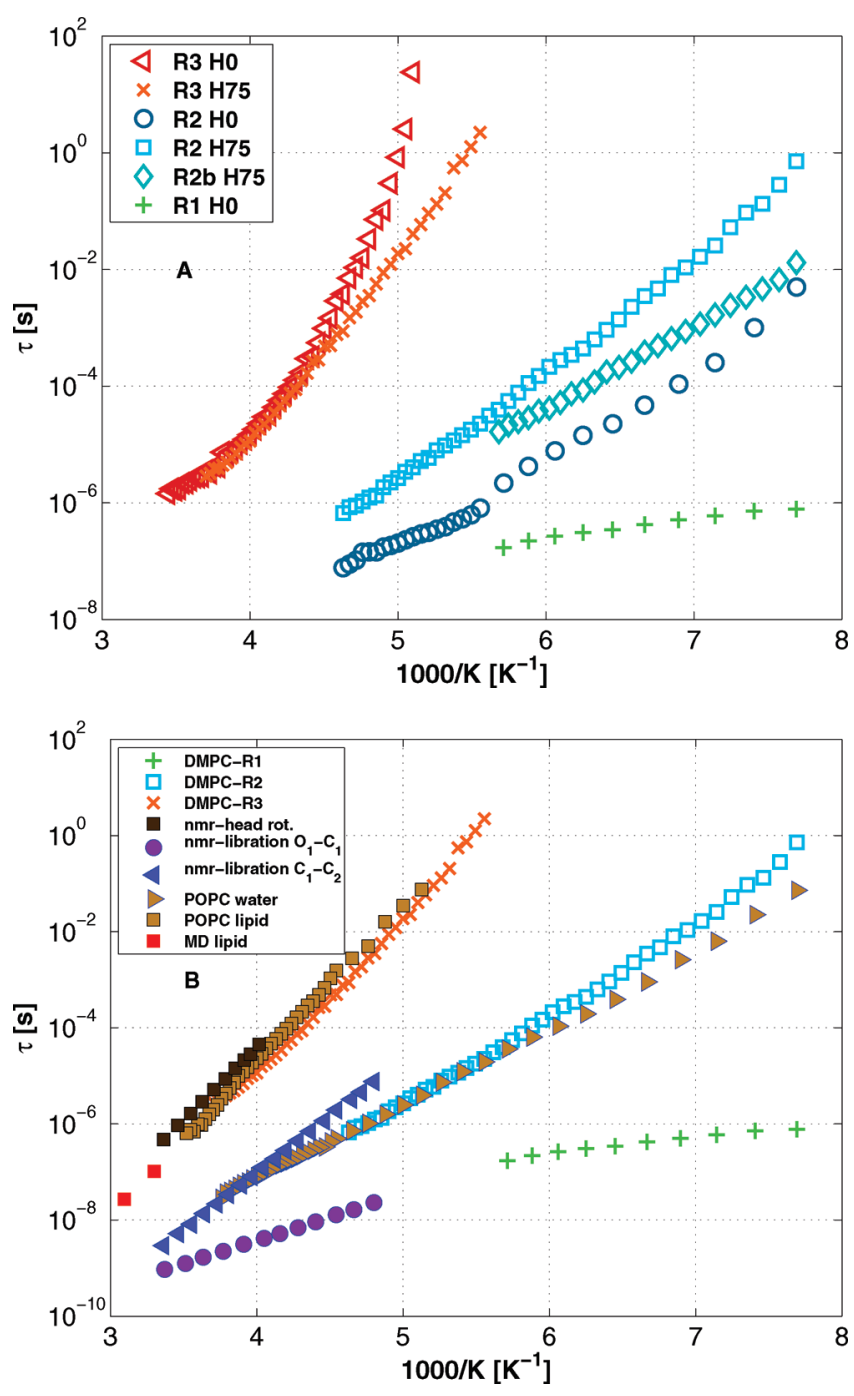

Figure 5. (A) Dielectric relaxation times of water (R1, R2) and lipid (R3) at low (H0) and intermediate (H75) hydration levels. (B) Comparison of lipid and water relaxation times from this work with ${ }^{31} \mathrm{P}$ NMR relaxation times ${ }^{43}$ and relaxation times from molecular dynamics (MD) simulations. ${ }^{2}$ In addition, we show dielectric relaxation times of palmitoyloleoyl phosphatidylcholine (POPC) bilayers. ${ }^{42}$ For locations of the NMR motions, see Figure 1.

local environments, as discussed below. Also, R1 is most likely a result of local water dynamics, as a similar process has been observed in other water containing systems. ${ }^{41}$

The main water relaxation (R2) has a relaxation time that is best described by a VFT temperature dependence (see Figure 4), indicating that the relaxation process has a cooperative behavior. Such a super-Arrhenius temperature dependence is generally not observed for supercooled interfacial water, ${ }^{30}$ since it can be considered as a local $\beta$-like relaxation process, at least in the temperature range $<180 \mathrm{~K}^{27}$ Thus, in most types of host materials, the supercooled interfacial water exhibits a noncooperative thermally activated relaxation process, which, furthermore, is slower than the here observed main water relaxation, as shown in Figure 6. Therefore, we suggest that the R2 process involves water molecules which are influenced by fast local lipid motions, such as the libration around $\mathrm{O}_{1}-\mathrm{C}_{1}$ in Figure 1, as observed by $\mathrm{NMR}^{43}$ (see Figure 5B). The coupling to the local lipid dynamics may both speed up the water dynamics as well as

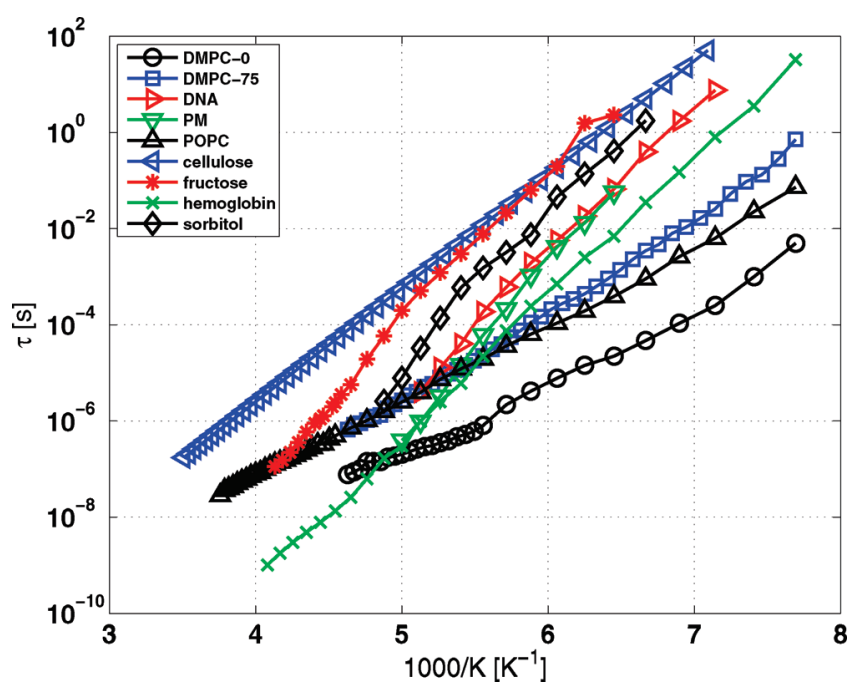

Figure 6. Dielectric relaxation times of supercooled and glassy water in a wide variety of systems: DMPC (this work), POPC, ${ }^{42}$ purple membrane (PM), ${ }^{21} \mathrm{DNA}^{30}{ }^{30}$ hemoglobin, ${ }^{51}$ sorbitol, ${ }^{30}$ fructose, ${ }^{30}$ and cellulose. $^{29}$

alter its intrinsic Arrhenius temperature dependence due to a temperature dependent coupling.

The process denoted $\mathrm{R} 2 \mathrm{~b}$ is only observed in the two hydrated samples H75 and H84, as shown in Figure 4. It is attributed to a similar type of local water relaxation as R2, but of water molecules with a different coordination to the lipid molecules. In fact, studies with infrared spectroscopy show evidence for three distinct hydrogen bonded water motifs in phospholipid membrane samples with zero, one, or both $\mathrm{OH}$ moieties hydrogen bonded. ${ }^{48}$ Since the temperature dependence of $\mathrm{R} 2 \mathrm{~b}$ is given by the Arrhenius law with an activation energy of $0.28 \mathrm{eV}$, which roughly corresponds to the energy required to break one hydrogen bond, we can expect that these water molecules have only one of their two $\mathrm{OH}$ moieties hydrogen bonded. This activation energy can also be compared with the activation energy of $0.54 \pm 0.04 \mathrm{eV}$ (corresponding to the breaking of two hydrogen bonds) of the general intrinsic water relaxation of supercooled interfacial water, ${ }^{30}$ shown in Figure 6 for some important classes of biological molecules as sugars and proteins. Thus, the activation energy of $\mathrm{R} 2 \mathrm{~b}$ is about half of the value for the intrinsic water relaxation, indicating the involvement of less hydrogen bonded water molecules. The interpretation that these water molecules are less hydrogen bonded is also supported by the finding that $\mathrm{R} 2 \mathrm{~b}$ cannot be observed in the dried sample $\mathrm{HO}$, where all water molecules should be strongly interacting with the lipid molecules and therefore be most influenced by the fast local lipid motions. The fact that R2b has merged with R2 at temperatures above $200 \mathrm{~K}$ suggests that the water molecules become less localized at higher temperatures, and therefore less influenced by a specific local lipid environment.

In Figure 5A, process R2 is compared for the two hydration levels $\mathrm{HO}$ and H75. From this figure, it is evident that the main water relaxation is substantially faster in the dried $\mathrm{HO}$ sample than in the H75 (and H84, not shown) samples. The same behavior was observed in a recent dielectric study by Tielrooj et al., ${ }^{49}$ where they also concluded that water molecules with less hydrogen bonds than in bulk water, due to the interaction with lipid headgroups, relax faster than water molecules in a bulk-like environment. This behavior is in direct contrast to the normal 
behavior of supercooled interfacial water, where the relaxation time decreases substantially with increasing size of the water clusters, and thereby also with decreasing interaction with the host material. ${ }^{50}$ The anomalous speeding up of the water dynamics in the dried $\mathrm{H0}$ sample is another indication (in addition to the super-Arrhenius temperature dependence and the fact that these water molecules, at least in the $\mathrm{HO}$ sample, should be strongly interacting with the lipid molecules) of the fact that this relaxation process is more determined by fast local lipid motions than by the intrinsic water dynamics.

\section{- CONCLUSIONS}

The results presented in this paper demonstrate the strong interplay between the hydration water and the lipid headgroup dynamics. It is clear that the lipid headgroup rotation is influenced by the hydration water and that the water dynamics is influenced by faster and more local lipid headgroup dynamics. Lowering the water content in lipid bilayer systems results in a reduced space between the lipid molecules, which increases the intermolecular lipid correlations and thereby also the length scale of cooperativity of the lipid headgroup dynamics. This increased cooperativity shows up as an increasing super-Arrhenius temperature dependence of the dielectric lipid headgroup rotation with decreasing hydration level. A reduced water content is also affecting the water dynamics but not in the normal way. With a reduced amount of water, less water molecules participate in a normal $\mathrm{H}$-bonding network and instead the interaction with the surrounding material increases. This surface interaction generally slows down the water dynamics, but in lipid membranes where fast local lipid dynamics is present, the motion of the interacting water molecules actually speeds up. Thus, at low hydration levels, the water relaxation becomes more determined by fast local lipid motions than by the intrinsic water dynamics. Finally, our experimental results suggest that lipid bilayer systems may exhibit similarities with colloidal systems regarding the glass transition related dynamics, since in both systems an increased softness results in a "stronger" glass-forming behavior.

\section{AUTHOR INFORMATION}

\section{Corresponding Author}

*E-mail: peter.berntsen@chalmers.se (P.B.); jan.swenson@ chalmers.se (J.S.). Phone: +46 3177256 80. Fax: +46 31772 2090.

\section{ACKNOWLEDGMENT}

We thank Silvina Cerveny and Helén Jansson for the experimental data shown in Figure 6. This work was financially supported by the Swedish Research Council and the Swedish Energy Agency.

\section{REFERENCES}

(1) Milhaud, J. Biochim. Biophys. Acta, Biomembr. 2004, 1663, 19-51.

(2) Högberg, C.-J.; Lyubartsev, A. P. J. Phys. Chem. B 2006, 110, $14326-14336$.

(3) Semrau, S.; Idema, T.; Holtzer, L.; Schmidt, T.; Storm, C. Phys. Rev. Lett. 2008, 100, 088101.

(4) Lee, H. J.; Peterson, E. L.; Phillips, R.; Klug, W. S.; Wiggins, P. A. Proc. Natl. Acad. Sci. U.S.A. 2008, 105, 19253-19257.

(5) Crowe, J. H.; Crowe, L. M. Nat. Biotechnol. 2000, 18, 145-146.

(6) Trounson, A.; Mohr, L. Nature 1983, 305, 707-709.
(7) Rand, R. P.; Parsegian, V. A. Biochim. Biophys. Acta, Rev. Biomembr. 1989, 988, 351-376.

(8) Gaede, H. C.; Gawrisch, K. Biophys. J. 2003, 85, 1734-1740.

(9) Fitter, J.; Lechner, R.; Dencher, N. J. Phys. Chem. B 1999, 103, 8036-8050.

(10) Doxastakis, M.; Sakai, V. G.; Ohtake, S.; Maranas, J.; de Pablo, J. Biophys. J. 2007, 92, 147-161.

(11) Swenson, J.; Kargl, F.; Berntsen, P.; Svanberg, C. J. Chem. Phys. 2008, 129, 045101.

(12) Hildenbrand, M.; Bayerl, T. Biophys. J. 2005, 88, 3360-3367.

(13) Anotonietti, M.; Neese, M.; Blum, G.; Kremer, F. Langmuir 1996, 12, 6636-4441.

(14) Haibel, A.; Nimtz, G.; Pelster, R.; Jaggi, R. Phys. Rev. E 1998, 57, $4838-4841$.

(15) Klösgen, B.; Reichle, C.; Kohlsmann, S.; Kramer, K. D. Biophys. J. 1996, 71, 3251-3260.

(16) Pottel, R.; Göpel, K.-D.; Henze, R.; Kaatze, U.; Uhlendorf, V. Biophys. Chem. 1984, 19, 233-244.

(17) Cooper, J.; Hill, R. J. Colloid Interface Sci. 1996, 180, 27-35.

(18) Tirado, M.; Grosse, C.; Schrader, W.; Kaatze, U. J. Non-Cryst. Solids 2002, 305, 373-378.

(19) Enders, A.; Nimtz, G. Ber. Bunsen-Ges. Phys. Chem. 1984, 88, 512-517.

(20) Raudion, A.; Castelli, F.; Briganti, G.; Cametti, C. J. Chem. Phys. 2001, 115, 8238-8250.

(21) Berntsen, P.; Bergman, R.; Jansson, H.; Weik, M.; Swenson, J. Biophys. J. 2005, 89, 3120-3128.

(22) Bruning, B.; Wald, E.; Schrader, W.; Behrends, R.; Kaatze, U. Soft Matter 2009, 5, 3340-3346.

(23) Rog, T.; Murzyn, K.; Pasenkiewicz-Gierula, M. Chem. Phys. Lett. 2002, 352, 323-327.

(24) Tieleman, D.; Marrink, S.; Berendsen, H. Biochim. Biophys. Acta 1997, 1331, 235-270.

(25) Lopez, C. F.; Nielsen, S. O.; Klein, M. L.; Moore, P. B. J. Phys. Chem. B 2004, 108, 6603-6610.

(26) Koynova, R.; Caffrey, M. Biochim. Biophys. Acta, Rev. Biomembr. 1998, 1376, 91-145.

(27) Swenson, J.; Jansson, H.; Bergman, R. Phys. Rev. Lett. 2006, 96, 247802.

(28) Buchsteiner, A.; Lechner, R. E.; Hauss, T.; Dencher, N. A. J. Mol. Biol. 2007, 371, 914-923.

(29) Cerveny, S.; Schwartz, G. A.; Bergman, R.; Swenson, J. Phys. Rev. Lett. 2004, 93, 245702.

(30) Cerveny, S.; Alegría, A.; Colmenero, J. Phys. Rev. E 2008, 77, 031803.

(31) Huang, D. M.; Chandler, D. Proc. Natl. Acad. Sci. U.S.A. 2000, 97, 8324-8327.

(32) Daidone, I.; Ulmschneider, M. B.; Di Nola, A.; Amadei, A.; Smith, J. C. Proc. Natl. Acad. Sci. U.S.A. 2007, 104, 15230-15235.

(33) Seul, M.; Sammon, M. Thin Solid Films 1990, 185, 287-305.

(34) Greenspan, L. J. Res. Natl. Bur. Stand., Sect. A 1977, 81A, 89.

(35) Faure, C.; Bonakdar, L.; Dufourc, E. J. FEBS Lett. 1997, 405, 263-266.

(36) Verdonck, E.; Schaap, K; Thomas, L. Int. J. Pharm. 1999, 192, 3-20.

(37) Vogel, H. Phys. Z. 1921, 22, 645-646.

(38) Fulcher, G. J. Am. Ceram. Soc. 1925, 8, 339-355.

(39) Tamman, G.; Hesse, G. Z. Anorg. Allg. Chem. 1926, 156, $245-$ 257.

(40) Böhmer, R.; Ngai, K. L.; Angell, C. A.; Plazek, D. J. J. Chem. Phys. 1993, 99, 4201.

(41) Swenson, J.; Jansson, H.; Hedström, J.; Bergman, R. J. Phys.: Condens. Matter 2007, 19, 205109.

(42) Svanberg, C.; Berntsen, P.; Johansson, A.; Hedlund, T.; Axen, E.; Swenson, J. J. Chem. Phys. 2009, 130, 035101.

(43) Dufourc, E. J.; Mayer, C.; Stohrer, J.; Althoff, G.; Kothe, G. Biophys. J. 1992, 61, 42-57.

(44) Trapp, M.; Gutberlet, T.; Juranyi, F.; Unruh, T.; Demé, B.; Tehei, M.; Peters, J. J. Chem. Phys. 2010, 133, 164505. 
(45) Petrache, H. I.; Gouliaev, N.; Tristram-Nagle, S.; Zhang, R.; Suter, R. M.; Nagle, J. F. Phys. Rev. E 1998, 57, 7014-7024.

(46) Mattsson, J.; Wyss, H. M.; Fernandez-Nieves, A.; Miyazaki, K.; Hu, Z.; Reichman, D. R.; Weitz, D. A. Nature 2009, 462, 83-86.

(47) Shalaev, E. Y.; Zografi, G.; Steponkus, P. L. J. Phys. Chem. B 2010, 114, 3526-3533.

(48) Volkov, V.; Palmer, D.; Righini, R. Phys. Rev. Lett. 2007, 99, 078302 .

(49) Tielrooij, K. J.; Paparo, D.; Piatkowski, L.; Bakker, H. J.; Bonn, M. Biophys. J. 2009, 97, 2484-2492.

(50) Sjöstrom, J.; Mattsson, J.; Bergman, R.; Johansson, E.; Josefsson, K.; Svantesson, D.; Swenson, J. Phys. Chem. Chem. Phys. 2010, 12, 10452-10456.

(51) Jansson, H. Unpublished data. 